\title{
Un scriptorium et son époque : les chanoines de Beauport et la société bretonne au Moyen-Âge
}

Jean-René Ladurée

\section{Q OpenEdition \\ 1 Journals}

Édition électronique

URL : http://journals.openedition.org/abpo/3743

DOI : $10.4000 / a b p o .3743$

ISBN : 978-2-7535-7411-3

ISSN : $2108-6443$

\section{Éditeur}

Presses universitaires de Rennes

Édition imprimée

Date de publication : 20 décembre 2017

Pagination : 194-198

ISBN : 978-2-7535-7409-0

ISSN : 0399-0826

\section{Référence électronique}

Jean-René Ladurée, «Un scriptorium et son époque : les chanoines de Beauport et la société bretonne au Moyen-Âge », Annales de Bretagne et des Pays de l'Ouest [En ligne], 124-4 | 2017, mis en ligne le 20 décembre 2017, consulté le 23 septembre 2020. URL : http://journals.openedition.org/abpo/3743 ; DOI : https://doi.org/10.4000/abpo.3743

Ce document a été généré automatiquement le 23 septembre 2020.

(C) Presses universitaires de Rennes 


\title{
Un scriptorium et son époque : les chanoines de Beauport et la société bretonne au Moyen-Âge
}

\author{
Jean-René Ladurée
}

\section{RÉFÉRENCE}

JEANNEAU, Cédric (dir.), Un scriptorium et son époque : les chanoines de Beauport et la société bretonne au Moyen-Âge, Centre de Recherche Bretonne et Celtique, Université de Bretagne Occidentale, Brest, 2015, 492 p.

1 Le Centre de Recherche Bretonne et Celtique peut s'enorgueillir d'avoir publié un riche et volumineux ouvrage de près de 500 pages sur l'unique abbaye prémontrée de Bretagne: Beauport. Sous la direction de Cédric Jeanneau, maître de conférences d'Histoire médiévale à l'Université de Bretagne Occidentale, l'ensemble se présente comme la mise en forme d'un colloque international tenu les 13 et 14 juin 2013 à Brest et sur le site même de l'abbaye. Il ambitionne d'embrasser toute l'histoire de cette structure costarmoricaine depuis sa fondation en 1202 par le comte de Goëlo, Alain Ier d'Avaugour et son épouse, jusqu'au xIXe siècle, malgré une couverture particulièrement dense pour le Moyen Âge. Doit-on préciser que la grande valeur de cet ouvrage réside dans la pluridisciplinarité de ses 16 contributeurs français et internationaux: historiens, archéologues, historiens d'art, linguistes, philologues et un géologue permettent de renouveler les connaissances sur ce lieu finalement méconnu; leur œuvre s'intègre pleinement dans le programme débuté en 2012 qui vise à collecter, puis à éditer les actes médiévaux de cette abbaye, ainsi que dans les multiples rencontres qui se sont déroulés sur ce sujet depuis 2013, notamment à Leeds et à Kalamazoo.

2 L'introduction du Père Bernard Ardura permet de poser les termes génériques du sujet. Qu'est-ce qu'un chanoine? Quelles sont les particularités de la Règle de SaintAugustin? Après quoi, l'ouvrage est divisé en 5 parties, somme toute assez 
logiquement. Elles correspondent globalement à l'organisation du colloque, même si certaines contributions transversales ont été transférées d'une thématique à une autre.

La première d'entre elles intitulée "une abbaye au miroir des sources » doit être considérée comme une indispensable entrée en matière (p. 27-84): l'organisateur du colloque reprend dans un premier temps les anciennes éditions du cartulaire de l'abbaye, notamment le travail déjà réalisé au xixe siècle par Jules Geslin de Bourgogne et Anatole de Barthelemy. Tout en montrant le mérite d'une telle édition, l'auteur fait le point sur le dossier documentaire qui s'avère plus riche encore et le colloque est alors l'occasion de faire le point sur l'avancée des travaux de la nouvelle édition. 510 chartes originales ont été inventoriées depuis la fondation à 1305. Les deux autres contributions rappellent quant à elles que la documentation diplomatique n'est pas la seule source exploitable. Amandine Le Goff fait notamment appel à des obituaires - en tout état de cause des extraits postérieurs notamment tirés des riches collections de Gaignières - afin de restituer assez classiquement les réseaux de fidélités gravitant autour de l'abbaye, quand André-Yves Bourgès revient pour sa part sur la promotion de certains saints bretons comme saint Josse au travers d'œuvres proprement littéraires composées par les chanoines de Beauport. Cette contribution est probablement la plus originale et la plus déroutante pour le chercheur, même si l'auteur reconnaît lui-même que le sujet n'est pas original, contrairement au regard nouveau qu'il porte sur celui-ci, distinguant l'impact du rôle de promotion des chanoines sur les saints de la tradition et sur les novi sancti.

4 Un second axe rebaptisé pour la publication «Beauport: une abbaye en Goëlo» regroupe quatre contributions qui ont comme point commun de replacer l'abbaye dans le contexte humain, social, politique et économique de la Bretagne essentiellement durant son premier siècle d'existence (p. 85-200). Yannick Hillion, à l'image des travaux cartographiques anciens de chercheurs comme André Dufief, réalise d'abord, une présentation de « la situation monastique de la Bretagne jusqu'en 1202 » rappelant que les chanoines de Beauport s'installent dans un espace déjà densément occupé par d'autres ordres monastiques, notamment des Bénédictins, des Cisterciens et des chanoines réguliers. L'existence de cette concurrence spirituelle n'est pas sans conséquences sur les rapports avec les généreux donateurs du Goëlo. Assez classiquement, l'étude des liens sociaux réalisée par Yves Coativy attribue la place centrale dans la démarche de dotations et de donations aux familles aristocratiques locales à la recherche des prières mémorielles et d'un lieu de sépulture. Il est difficile de s'installer sur un territoire déjà occupé, même si les chanoines surent se démarquer par la desserte des églises, augmentant par là même leur présence au sein des communautés villageoises. C'est en prenant en compte ce cadre que le directeur de cette publication a inséré sa seconde intervention: les chanoines recevaient majoritairement des terres, et aussi des dîmes, mais peu de droits seigneuriaux, il leur fallut donc mener une véritable réflexion sur la mise en valeur de leur domaine, «bâtir des granges, acquérir de nouvelles parcelles pour renforcer leur emprise foncière " (p. 88). En somme, Cédric Jeanneau vient confirmer un pan assez attendu de l'histoire économique d'une abbaye au Moyen Âge. C'est Paul Evans, de l'université de York, qui clôt cette partie avec l'une des contributions les plus originales de cet ouvrage collectif: "Histoires d'argent: emprunts et prêts dans les chartes" où il évoque l'épineux sujet du prêt par les structures monastiques. L'abbaye de Beauport prête de l'argent sur la base de contrats pignoratifs (29 sont étudiés) durant une période restreinte allant de 1226 à 1279 . L'appel de la croix apparaît comme un élément majeur 
de cette activité, mais après 1270 et la dernière croisade, cela ne suffit plus pour comprendre l'attrait du prêt canonial. À compter de cette date, et pour une très courte période, la sociologie des emprunteurs et la valeur des sommes empruntées deviennent en effet beaucoup plus modestes et révèlent une tout autre réalité économique. Encore faut-il se garder de considérations trop générales, car le contributeur, reprenant à son compte la thèse d'André Chevdeville, rappelle que des arrangements n'ont certainement pas été mis par écrit, permettant alors aux chanoines d'utiliser ces contrats pignoratifs à des fins usuraires.

Une partie essentielle, «Une abbaye gothique au cœur du Goëlo », qui concentre trois contributions strictement complémentaires, rendra de nombreux services aux historiens d'art soucieux de mieux connaître l'histoire d'un bâti monastique ayant inspiré de nombreux artistes (p. 201-264). Citant saint Bernard, Louis Chauris, grand spécialiste de la lithologie des édifices monastiques bretons et géologue de profession, se penche d'abord sur la question des matériaux pierreux ayant servi à la construction et à la restauration de l'abbaye de Beauport. Il démontre merveilleusement bien que dès les origines, des roches locales - spilites vert-violacé, schistes bleus, grès roses et pierre verte - de même que des pierres d'approvisionnement plus régional comme le granite ocre ou le granite blanc-gris ont été sélectionnées depuis plusieurs carrières parfois très éloignées de l'abbaye, ce qui a pour incidence de créer ce que l'auteur appelle "un véritable polylithisme coloré des façades». La provenance de certaines pierres est à remarquer : ainsi, fait-on très probablement appel dès le xiIIe siècle au réputé calcaire de Caen, même si ce dernier prend une importance plus cruciale au xve siècle, notamment avec la reconstruction du réfectoire. La périodisation est également essentielle. L'auteur nous apprend ainsi que le granite de l'île-Grande ne semble pas utilisé avant le xvire siècle, lorsque la «salle au Duc » est rehaussée. AnneClaude Ballini, présidente des Amis de Beauport, se charge quant à elle de faire le point sur les différentes campagnes de fouilles menées entre 1992 et 2012, ce qui permet d'actualiser les connaissances des chercheurs sur les étapes de construction et d'aménagements du site, notamment la complexité du réseau hydraulique conçu par les chanoines. La proximité à la mer forme un atout pour les chanoines, mais la lutte contre l'invasion des eaux salées explique également la construction de différents dispositifs dès le xiIIe siècle comme la digue nommée «le talus de Beauport ». L'auteur évoque, images à l'appui, les multiples découvertes faites suite soit à des travaux, soit à des fouilles programmées : carrelages, faïences, sculptures, enduits et décors peints l'ensemble s'étalant du xiIIe au xixe siècle - sont ainsi présentés. De manière complémentaire, Yves Gallet, maître de conférences à l'Université de Bretagne Occidentale, évoque la question de la circulation dans l'abbaye et sa distribution fonctionnelle. L'historien d'art médiéval revisite complètement les connaissances et les interprétations sur ce sujet en évoquant successivement «le degré d'élaboration du programme sculpté, la qualité du système de voûtement ou la diversité des roches mises en œuvre » (p. 203-204). Il distingue ainsi la voûte d'arcade, qui renvoie selon lui à des espaces de services, et la voûte d'ogive qui indiquerait un rang supérieur des bâtiments qui en sont dotés. Il rappelle également que le polylithisme déjà évoqué ne serait pas le résultat des contraintes d'approvisionnement, mais plutôt une volonté réfléchie des constructeurs, afin de distinguer l'importance et la valeur des espaces. Dans la pratique, il reprend une bonne part des conclusions d'un article qu'il avait publié en 2011 aux éditions Brepols. 
6 Trois rédacteurs prennent alors le relais pour envisager l'histoire de l'abbaye dans le temps long, notamment en évoquant les tumultes de l'époque moderne (p. 265-366). Les guerres de religion et les dissensions politiques du xvie siècle ont mis fin à l'extraordinaire prospérité de Beauport, mais l'arrivée d'un nouveau prieur conventuel dans les années 1660-1690, Vincent Royer, un «visionnaire » (p. 270) dans un contexte plus global de réforme et de modernisation de la liturgie et de la spiritualité, va participer au renouveau de celle-ci. Harriet M. Sorre de Torrens évoque l'empreinte de ce prieur sur la restauration matérielle et spirituelle de l'abbaye sous le prisme de la commande de plusieurs retables aujourd'hui disparus. Dès 1669, V. Royer s'adresse à de réputés architectes lavallois pour faire réaliser trois retables : d'abord Olivier Martinet, puis une décennie plus tard Michel Guérin. Les contrats de ces artisans, des fragments archéologiques, ainsi que des ouvrages de J.-C. Van de Sterre et de V. Royer lui-même permettent à l'auteur de cet article de proposer une reconstitution fidèle du premier retable. Il en ressort que le programme iconographique reflète les pratiques liturgiques des Prémontrés avec un rôle central joué par la Vierge Marie et les saints Norbert et Augustin, respectivement fondateur de l'Ordre et initiateur de la règle suivie par les chanoines. S'ensuit une contribution plus synthétique de Samuel Gicquel sur celui que l'on peut considérer comme le dernier chanoine de Beauport: Hervé-Julien Le Sage (1757-1832), alors même que la Révolution a mis un point d'arrêt final à la vie monastique à Beauport. L'auteur, maître de conférences à l'université Rennes 2, revient sur les Mémoires historiques du chanoine qu'il connaît très bien. Ces mémoires ne forment pas une autobiographie de son rédacteur, mais elles sont l'occasion de décrire ce qu'il considère être les excès de son temps et intrinsèquement les erreurs à ne pas répéter par opposition au " paradis perdu » que représentait la quiétude idéalisée et paradoxale de sa vie à Beauport. Malgré sa vente comme bien national en 1790, l'histoire de l'abbaye continue de s'écrire au xIxe siècle, mais ce récit prend des formes poétique, artistique et légendaire. Sophie Gondolle, docteur en littérature évoque successivement la difficulté à connaître les origines de l'abbaye, faute de traces historiques suffisantes, et les témoignages laissés par des archéologues et des antiquaires comme le chevalier de Frémenville. Elle poursuit avec la contribution des voyageurs à la constitution d'un tableau pittoresque de l'abbaye, puis elle rappelle que de nombreuses croyances, transmises oralement puis mises par écrit, sous-entendent l'existence d'un trésor et d'un souterrain qui ont pour simples origines la découverte d'une cache, puis d'un escalier que même le chanoine Le Sage ne connaissait pas en son temps. Des auteurs locaux se sont aussi saisis des mythes de Beauport pour créer leurs propres récits fantastiques et de nombreux contes évoquent l'exécrable réputation des chanoines, "mauvais riches" (p.345) et paresseux. Elle termine sa présentation en accordant une place non négligeable au témoignage écrit et iconographique laissé par le célèbre Anatole Le Braz et le fonds déposé dans les archives du CRBC.

L'ouvrage se clôt par l'évocation « des chanoines en Bretagne et ailleurs » (p. 367-466). Martine Plouvier propose de replacer les connaissances sur l'abbaye de Beauport dans le réseau médiéval prémontré des différentes circaries du "grand Ouest » en comparant les circonstances de fondation, les plans mis en place et l'architecture privilégiée. Les deux dernières contributions traitent spécifiquement de l'histoire de deux abbayes. La pénultième intervention de $\mathrm{C}$. Jeanneau concerne la première, à savoir Lieu-Dieu en Jard, abbaye du Bas-Poitou mais ayant des liens avec la Bretagne. Sa position en bordure de mer et la maîtrise d'un cabotage forment des points de similitudes avec Beauport. La seconde abbaye, Saint-Pierremont, fondation érémitique 
installée non loin de Briey en Lorraine, est étudiée par Yoric Schleef qui explique que l'importance du fonds d'archives lui a permis d'effectuer des parallèles avec Beauport dans les domaines du développement et de l'organisation des temporels et des liens avec les hommes qui peuplent la région. "Conservatisme » et «contemplation » sont les deux termes qui résumeraient le mieux cette fondation.

8 Le lourd travail de conclusion de l'ouvrage revient une nouvelle fois à celui qui en a dirigé la publication, C. Jeanneau, qui précise avec une grande sagesse : «l'historien... sait que rien n'est jamais définitif et que les recherches sur Beauport et les Prémontrés continuent...» 